\title{
Designing a Brain-Computer Interface controlled video-game using consumer grade EEG hardware
}

\author{
Marijn van Vliet, Arne Robben, Nikolay Chumerin, Nikolay V. Manyakov, Adrien Combaz \\ and Marc M. Van Hulle \\ Laboratorium voor Neuro- en Psychofysiologie \\ K.U. Leuven, Campus Gasthuisberg, Herestraat 49, B-3000 Leuven, Belgium \\ Tel: +32 16345640 , Fax: +32 16345993 \\ \{Nikolay.Chumerin,NikolayV.Manyakov,Adrien.Combaz,Arne.Robben,Marijn.vanVliet,Marc.VanHulle\}@ med.kuleuven.be
}

\begin{abstract}
-
This paper describes a first attempt to a brain-computer interface (BCI), created in a research lab and running on expensive equipment, to consumer grade equipment, allowing one to reach a much broader audience and raise public awareness of this new technology. With the BCI, the user can perform a complicated task through mind control only: playing a tactical video-game. Reliable control is accomplished by adapting a Steady-State Visual Evoked Potential (SSVEP) classifier to be robust enough to cope with the signal quality of the consumer grade electroencephalography (EEG) device used, i.e. the Emotiv EPOC. The difference in performance of the game running on research grade EEG equipment versus the Emotiv EPOC is examined. The game was tested by a broad audience during a public event and was well received.
\end{abstract}

- Index Terms - brain-computer interfacing, electroencephalography, steady-state visual evoked potentials, Emotiv EPOC, games.

\section{INTRODUCTION}

Brain computer interfacing (BCI) has received widespread attention in recent years since it enables the user to control his/her environment from his/her brain activity directly, bypassing the need for speech, gestures, or any other form of muscular activity. BCIs are currently mostly considered for augmenting or re-establishing communication and motor control capabilities of patients suffering from severe brain or muscular disorders [1]. In addition, they open up alternative means of human-machine interaction that are also attractive for healthy users.

Currently, the main application area for BCIs for healthy users seems to be games. The novelty of this technology captures the imagination of the players and its limitations can be turned into a game challenge that a successful player has to overcome [2]. The industry has picked up on the trend and several companies now supply cheap electroencephalography (EEG) products. Systems that achieved a lot of media attention are those based on the NeuroSky ${ }^{1}$ device, for instance the 'Force trainer' 2 . It allows the player to raise and lower a ball by linking the strength of the alpha rhythm produced by the players to the rotational speed of a fan. Mind Flex, ${ }^{3}$ produced

\footnotetext{
${ }^{1}$ http://www.neurosky.com

${ }^{2} \mathrm{http}: / /$ company.neurosky.com/products/force-trainer

${ }^{3} \mathrm{http}: / /$ www.mindflexgames.com
}

by Mattel Inc., which is also based on the NeuroSky device, takes this concept a step further and adds a turning knob with which the player can control the position of the fan, allowing for 2-dimensional control of the ball. The goal is to guide the ball through an obstacle course. These commercial systems rely on measuring the user's alpha activity, as was first demonstrated in a game by Hjelm and Browall [3] called Brainball, where two players sit across a table with a ball in the middle. The game is won when the ball reaches the opponent, which is accomplished by relaxing. The ball would roll towards the one with the lowest alpha power.

Many BCI Games are developed by research groups [4], covering a wide range of paradigms and game play styles, that are potentially much more exiting than current commercial offerings. These games rely on techniques such as the detection and classification of the P300 [5], event related (de)synchronization (ERD/ERS) [6] and steady-state visual evoked potentials (SSVEP) [7]. These techniques offer precise control, allowing the user to issue explicit commands such as turn left and jump. During game play, it is immediately clear the system is at fault when it misinterprets an explicit command, as opposed to using measures of relaxation/concentration, where the player can be blamed for not being relaxed/concentrated enough. However, these games are mostly proofs of concept that rely on expensive research equipment that require the help of a second person to mount on the user and that are therefore cumbersome to demonstrate outside the lab.

In this paper, we try to bridge the gap between the commercial offerings and games developed by research groups, by creating a BCI that allows the user to operate a complicated, tactical game by issuing explicit commands, while running on cheap, consumer grade hardware. The game will rely on the detection and classification of the users SSVEP response, which means the user will control the game by focussing his/her eyes on different parts of the screen. The game is meant to be enjoyed by a large audience, taking into account the limitations of the technology, the cost of hard- and software, the ease of use, and the overall user experience. Foremost, the user must feel in control of the game. We formulated the following requirements for the game:

1) The target audience will be healthy users of all ages. 
2) The user will be able to control the game entirely with his/her brain activity. No other input methods should be required during game play.

3) The system will run robustly on consumer grade electroencephalography (EEG) hardware.

4) The user can monitor the output of the classifier and modify its parameters in real time.

The game was evaluated in both a lab setting and during a public event. In the lab setting, the difference in SSVEP classification performance on the signal of a research grade EEG system versus the Emotiv EPOC was examined. During the public event, only the EPOC was used and the game itself was evaluated.

\section{Methods}

The game that was developed is a variant of the 'tower defense' genre. The goal is to protect a tower against waves of enemies, who shall appear at one or more fixed points in the game world and walk towards the tower. When an enemy reaches the tower, the player loses the game. To prevent that, the user can build a limited amount of defensive structures. The user needs to decide on the optimal location of these defenses, based on information about the number of enemies that will appear at which positions. Because the game should be suitable for all ages (requirement 1), no violence is being shown: the enemies are giant red balls, which disappear upon being hit. A compilation from multiple screenshots is shown in figure 1, explaining the various elements of the game.

To control the game, the user needs some method to make a selection on the screen. Requirement 2 states that this should be possible by analyzing the user's brain activity alone. At the beginning of the level, the user makes a selection from several predefined locations to build defensive structures. When the user is satisfied with the layout, he/she can select the 'done' button, which will unleash the enemies. From that point on, the user loses control until either all enemies have been defeated, or an enemy reaches the tower and the user loses the game. An undo option is also available, which will undo the last build command, enabling the user to correct mistakes made by either himself or the system.

Three levels were designed. The first level is used while explaining the game mechanics to the user and is simply a straight line with the tower at one end and the enemies appearing at the other. The user cannot make strategical mistakes in this level. The other two levels require the user to think about where to place the defensives, making them harder and more interesting at the same time.

\section{A. Hardware and software}

The three EEG systems available for consumers on the market today that we are aware of, are the MindSet and MindWave, produced by NeuroSky, ${ }^{4}$ and the EPOC, produced by Emotiv. ${ }^{5}$ As the SSVEP is generated in the visual cortex,

\footnotetext{
${ }^{4}$ http://www.neurosky.com

${ }^{5}$ http://www.emotiv.com
}

we chose the EPOC, as it provides 14 channels, versus the one channel offered by both the MindSet and the MindWave, located on the forehead. The EPOC uses electrodes, dampened with a salt water solution, which in our experience can be set up in roughly 3 minutes by the user himself. This is a clear advantage over systems meant for research, which use gel electrodes and require a second person to set up, taking about 10 minutes.

To determine the difference in signal quality between the EPOC and a typical research system, we performed all experiments with the EPOC and with active $\mathrm{Ag} / \mathrm{AgCl}$ electrodes of the ActiCap system, developed by Brain Products, amplified by a prototype of an ultra low-power 8-channel wireless amplifier, developed by imec ${ }^{6}$.

For the project, only software tools that are available free of charge were used. The graphics and game logic are build using Unity $3 \mathrm{D},{ }^{7}$ a professional game engine, used by many of the smaller game companies. Both free and a commercial versions are available, with the commercial version offering more advanced capabilities. In this project, the free version was used. For signal recording and processing, Python $^{8}$ was used in combination with the NumPy and SciPy packages ${ }^{9}$, that are used for general numeric computation, and the GolemML/PsychicML packages ${ }^{10}$, that specialize in EEG analysis.

\section{B. SSVEP Stimulation}

Most currently available consumer systems for measuring brain activity use electroencephalography (EEG), where electrodes are placed on the scalp to record electrical activity caused by the firing of large groups of neurons.

One of the EEG potentials which can be robustly classified is the Steady State Visual Evoked Potential (SSVEP). When the user is focused on a visual stimulus that flickers at a sufficiently high rate $(f \geq 6 \mathrm{~Hz}$.) The individual transient visual responses overlap, resulting in a steady state signal, observable mostly in the occipital area [8]. When examining the Fourier transform of the signal, an increased amplitude can be seen at the same frequency as the stimulus and it's harmonics $(2 f, 3 f, \ldots)$

Many SSVEP-based systems allow the user to make a selection by presenting multiple stimuli that flicker at different frequencies [9]. The system detects the frequency of the SSVEP response and by that means at which stimulus the user is looking.

For our game, we decided to use a different method. Only one stimulus is presented at the bottom-left corner of the screen, flickering at a fixed frequency. The system detects whether the user is looking at the stimulus or not. The selection options are highlighted one by one, for 2 seconds each. When

\footnotetext{
${ }^{6}$ Interuniversity Microelectronics Centre (IMEC), http://www.imec.be

${ }^{7}$ http://www.unity3d.com

${ }^{8}$ http://www.python.net

${ }^{9}$ http://www.scipy.org

${ }^{10} \mathrm{http} / / /$ code.google.com/p/golemml, http://code.google.com/p/psychicml, developed by the BCI@HMI group at the University of Twente.
} 


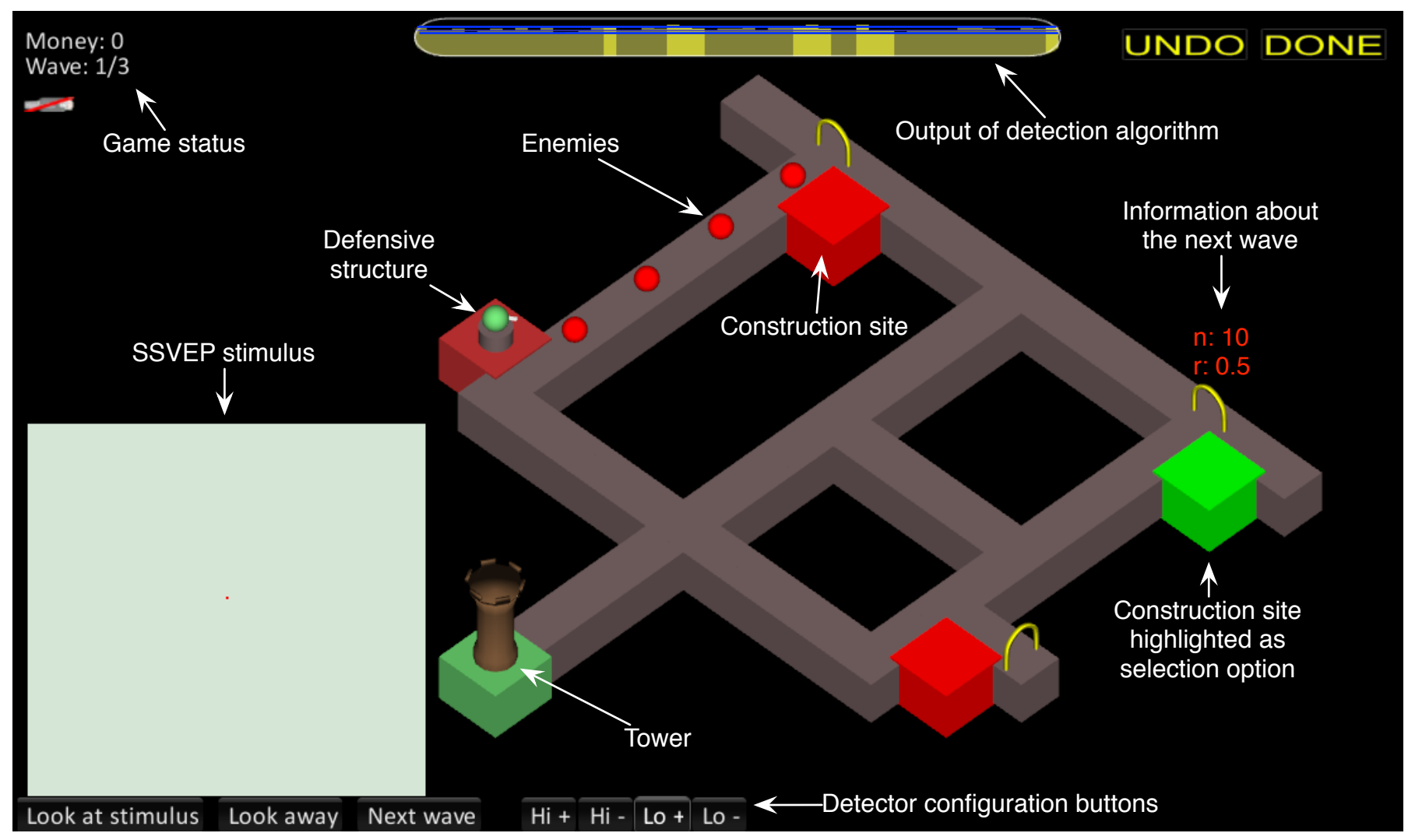

Fig. 1. Compilation from multiple screenshots showing all the elements of the game world and the interface.

the desired option is highlighted, the player looks at the flickering stimulus. When the system detects the presence of a SSVEP response, the currently highlighted option is selected.

The stimulus is a square with a width and height of approximately 10 . The color of the stimulus was modulated by mapping a sine wave with a frequency of $12.8 \mathrm{~Hz}$ to grayscale values, where 0 means completely black and 1 means completely white. A small red dot is shown in the middle of the stimulus, which users indicated helps to keep the eyes focussed.

To determine the optimal frequency for the stimulus, a small experiment was performed, where 4 subjects were shown a SSVEP stimulus, of which the frequency was increased from $6 \mathrm{~Hz}$ to $30 \mathrm{~Hz}$. Results showed that all subjects had a good SSVEP response between $10-20 \mathrm{~Hz}$. For the game, the stimulus was fixed at $12.8 \mathrm{~Hz}$, as it lies in the range of reliably detectable frequencies and avoids rounding errors when recording with one of the devices (the EPOC, see section II-A), which has a samplerate of $128 \mathrm{~Hz}$, resulting in a SSVEP signal with a period of exactly 10 samples (see section II-C).

To obtain some data to determine optimal threshold for the SSVEP detection algorithm (section II-C) and to determine its performance, a short calibration is performed at the beginning of the game. The user looks at the center of the screen where a fixation cross is shown for 5 seconds, followed by 10 seconds of a $12.8 \mathrm{~Hz}$ SSVEP stimulus (width and height 5), 10 seconds fixation cross, 10 seconds SSVEP stimulus and, finally, 10 seconds fixation cross.

\section{SSVEP detection algorithm}

To robustly detect the presence of a SSVEP signal, we adapted an approach by An Luo and Thomas Sullivan [10] called Stimulus-Locked Inter-trace Correlation (SLIC), which determines the presence of a SSVEP signal in the time domain, as opposed to traditional classifiers that work in the frequency domain [9]. See figure 2 for a visualization of the process outlined below.

The recorded EEG signal is bandpass filtered between 2$45 \mathrm{~Hz}$ using a fourth-order Butterworth filter and decomposed in independent components using the JADE algorithm [11]. The Independent Component (IC) decomposition matrix is determined using the calibration data and thereafter kept fixed during game play.

All of the resulting ICs are divided in windows of a user defined length $l_{w}$ seconds with a fixed overlap of 0.5 seconds. Each window is split into non-overlapping segments of length $l_{s}=r / f$ samples, where $r$ is the sample rate of the signal and $f$ is the frequency of the SSVEP stimulus. For example, for a signal sampled at $r=128 \mathrm{~Hz}$, a window with length 1.0 seconds and a stimulus frequency $f=12.8 \mathrm{~Hz}$, we have a segment length of $l_{s}=128 / 12.8=10$ samples, which means splitting the window in $\left\lfloor l_{w} / l_{s}\right\rfloor=\lfloor 128 \cdot 1.0 / 10\rfloor=$ 12 segments containing the following samples: [1-10], [11$20], \ldots$ When the start and end positions of the segments do 

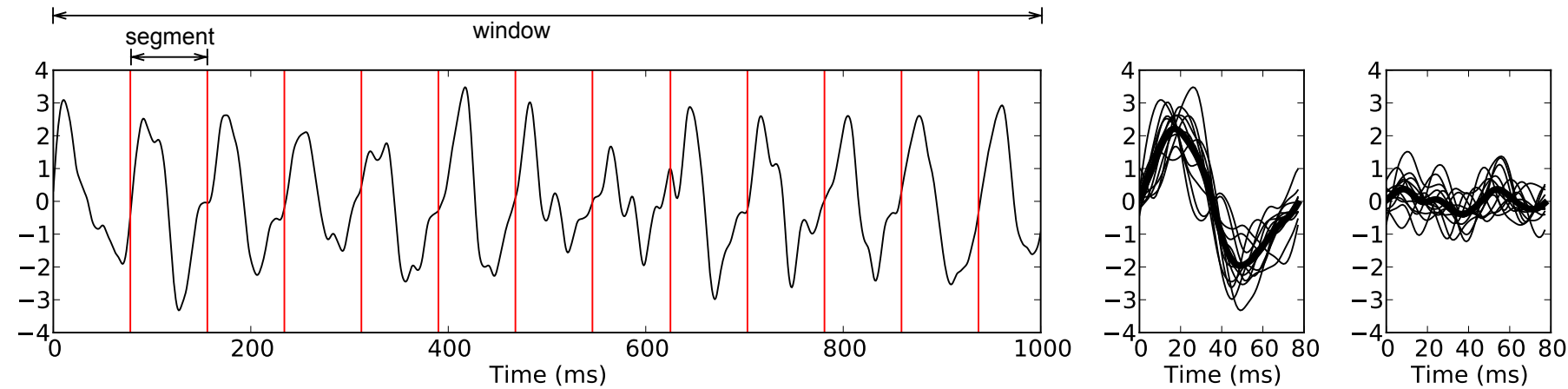

Fig. 2. Detection of a $12.8 \mathrm{~Hz}$ SSVEP signal, recorded by the IMEC device. Left: A one second window, subdivided into 12 segments and a remaining, incomplete segment which is discarded. The signal shown is not from a single electrode, but is one of the ICs resulting from the ICA step. Center: All extracted segments from the recording shown in the left panel. The mean is plotted as a thick line. Right: Segments extracted from a window where no SSVEP stimulus was shown, with the mean plotted as a thick line. Note that the correlation between the trials and the mean is much lower than those shown in the center plot.

not correspond to an exact number of samples as would for example be the case when using the IMEC device, which has a sampling rate of $1000 \mathrm{~Hz}$, the start positions are rounded to the nearest-integer value and the segment length is floored to the nearest-integer value. A sample rate of 1000 means a segment length of 78.125 , resulting in segments containing the following samples: [1-78], [79-156], ...

The splitting operation as described above yields an array W with a dimensionality of \#windows $\times$ \#ICs $\times$ \#segments $\times$ \#samples, iterated by $i, j, k$ and $l$ respectively. From this array, matrix $\mathbf{R}$ is constructed, which, for each window, and each IC, contains the likelihood of a SSVEP signal being present. To determine $\mathbf{R}$, the correlation coefficients between each segment and the average of all segments is calculated. The obtained correlation coefficients are themselves averaged to yield a single value between -1 and 1 , which is normalized to [0-1]. From matrix $\mathbf{R}$, vector $\mathbf{r}$, containing a single value for each window, is calculated by taking the maximum of each row of $\mathbf{R}$ :

$$
\begin{aligned}
\mathbf{R}_{i j} & =0.5+0.5 \cdot \underset{k}{\operatorname{mean}} \underset{l}{\operatorname{corr}}\left(\mathbf{W}_{i j k l}, \underset{m}{\operatorname{mean}} \mathbf{W}_{i j m l}\right) \\
\mathbf{r}_{i} & =\max _{j} \mathbf{R}_{i j} .
\end{aligned}
$$

The final step is to threshold the vector $\mathbf{r}$ using two threshold values $t_{h}$ and $t_{l}$. To determine these, the data collected during the calibration period were analyzed:

$$
\begin{aligned}
t_{h} & =\min \left(\operatorname{mean} \mathbf{s}, \frac{\operatorname{mean} \mathbf{s}+\max \mathbf{f}}{2}\right), \\
t_{l} & =\max \left(\operatorname{mean} \mathbf{f}, \frac{\operatorname{mean} \mathbf{f}+t_{h}}{2}\right) .
\end{aligned}
$$

Where $\mathbf{s}$ denotes the values of $\mathbf{r}$ during which the SSVEP stimulus was shown and $\mathbf{f}$ denotes the values of $\mathbf{r}$ where the subject was looking at a fixation cross. The thresholded version of $\mathbf{r}$, denoted $\mathbf{r}^{\prime}$, then becomes:

$$
\mathbf{r}_{i}^{\prime}=\left\{\begin{aligned}
0 & \text { if } i=0 \\
1 & \text { if } i>0 \text { and } \mathbf{r}_{i}>t_{h} \text { and } \mathbf{r}_{i-1}^{\prime}=0 \\
0 & \text { if } i>0 \text { and } \mathbf{r}_{i}<t_{l} \text { and } \mathbf{r}_{i-1}^{\prime}=1 \\
\mathbf{r}_{i-1}^{\prime} & \text { otherwise. }
\end{aligned}\right.
$$

Where $i$ iterates over each value of $\mathbf{r}$.

\section{RESUlTS}

To determine the performance of the detection algorithm, it was run on the calibration data. Eight users (aged 23-34, mean 26.75, std. 4.26, two female and six male) completed the calibration period with both the imec and the EPOC devices, before playing the game. The detected SSVEP periods were compared with the actual periods during which the SSVEP stimulus was shown (figure 3). For this offline analysis, the data were split into two parts, with the ICA and calculation of the threshold values being performed on the first part, and then applied to the second part, and vice versa. The percentage of correctly classified windows was used as a metric to compare the system using gel electrodes (the imec device) and the consumer grade system using salt water electrodes (the EPOC from Emotiv). The window size was increased from $0.5 \mathrm{~s}$ (containing only 6 periods of the $12.8 \mathrm{~Hz}$ stimulus) up to $2.5 \mathrm{~s}$. The windows are overlapping, with a window step of $0.5 \mathrm{~s}$.

The accuracy of the classifier increases with the window size, up to a certain point $( \pm 1.5 \mathrm{~s})$, after which the latency induced by the windowing operation counters the increase of classifier precision. From $1.5 \mathrm{~s}$ onwards, the imec device stops performing significantly better than the EPOC as determined by a two-tailed Wilcoxon signed rank test with testing criteria of $w \leq 4, p \geq 0.05$. For the game, window sizes of 1.0 s for the imec device and 1.5s for the EPOC were chosen as a good tradeoff between speed and accuracy.

Note that, during the game, each option is highlighted for $2 \mathrm{~s}$, a duration which corresponds to 10-15 windows, depending on the device used. Only one of them has to be classified as containing SSVEP in order to make the selection. The shown accuracy in the figure is therefore only useful to compare 

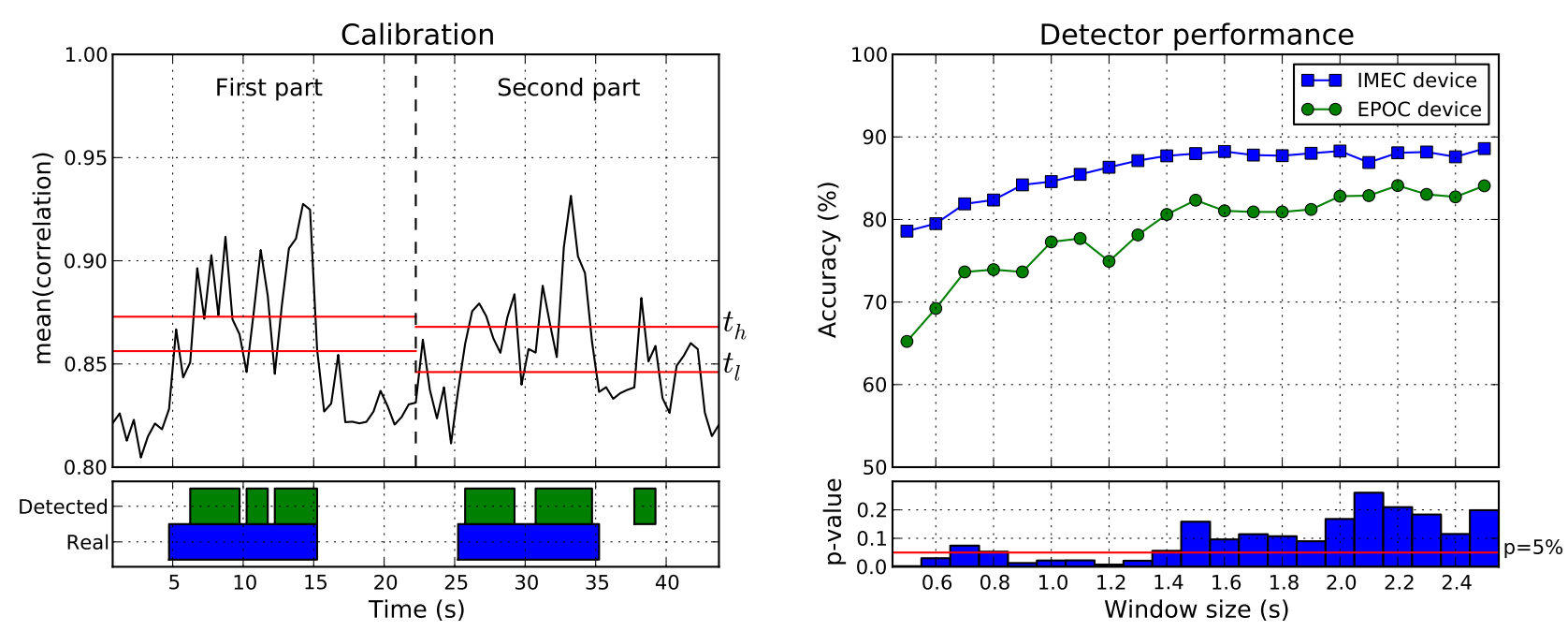

Fig. 3. Left: detection during calibration period using the EPOC device on a subject with average performance. Shown are the vector $\mathbf{r}$ along with the threshold values $t_{h}$ and $t_{l}$. Below are the detected periods of SSVEP activity along with the periods where the SSVEP stimulus was actually shown. The detector was trained on the first part and applied to the second part, and vise versa. In this configuration, a window size of $1.5 \mathrm{~s}$ was used and $85 \%$ of the windows were correctly classified. Right: performance of the detection algorithm for different window sizes during the calibration period (window step was fixed at $0.5 \mathrm{~s}$ ). Shown is the accuracy (\% windows correctly classified), averaged across 8 subjects, which all performed the calibration with both the imec and EPOC device. The $p$-values of a Wilcoxon signed rank test between the two devices is plotted at the bottom.

the performance of the two devices, but does not say much about the actual performance during the game. The in-game performance is considerably harder to quantify, as the user compensates for delays, and given that the thresholds can be tweaked. In this study, 7 users achieved proper control over the selection process and were able to complete all three levels. One user did not achieve control with any of the devices.

\section{A. Evaluation during public event}

During the I-Brain \& Senses event (18-19 March 2011, Ghent, Belgium), the game was tested in challenging conditions. Our booth stood in a noisy room, surrounded by wireless equipment. The users would wear the EPOC headset and play the game for a few minutes, talking to friends and moving around. In total, 25 users (ages 9-44, mean 22.88, std. 9.42, 13 male, 10 female) played the game with the EPOC device and filled in questionnaires afterwards.

Out of the 25 users, 9 easily achieved control, 13 found it challenging but achieved control and 3 users did not achieve any control at all. Upon being asked whether they found the game fun, 13 indicated 'yes' and 12 indicated 'not so much'. In the lab setting, the subjects would take some time to familiarize themselves with the selection method of looking at the SSVEP stimulus at exactly the right time. This and the complex game rules turned out to take too long to explain for an event during which users can only spend a few minutes with the game.

False positives in the detection are more frustrating than false negatives, since a false positive will make an unwanted selection, requiring use of the undo option, where a false negative will fail to make a selection, requiring the user to wait until after all other options are highlighted. A false positive on the done button is disastrous, as it cannot be undone and usually causes the user to fail the level. The user has some control over this by modifying the threshold values, which decreases the false positive rate at the expense of more false negatives.

Users quickly learned to compensate for the delay between looking at the stimulus and the formation of a detectable SSVEP signal, by anticipating which option will be highlighted next. Because they were able to monitor the detection process, and could instantly see the result of their actions, this was perceived as an enjoyable part of the game. Several users indicated that after a while, fixating on a SSVEP stimulus becomes tiring and will eventually become unpleasant.

\section{CONCLUSIONS}

Below, each requirement of the game will be discussed briefly:

1) The target audience will be healthy users of all ages. The game was tested on a wide age range (9-44) and while certainly not everyone enjoyed it, it did not depend on age. Children could grasp the game rules just as well as adults.

2) The user will be able to control the game entirely with his/her brain activity. No other input methods should be required during game play. The selection scheme allowed to the users to play the game without any conventional controls. Users mostly held their hands in their laps. The exception was the configuration interface, which uses a traditional GUI, but is not part of the game play.

3) The system will run robustly on consumer grade electroencephalography (EEG) hardware. In a lab setting, all users but one achieved control with both the imec device, which uses 8 active gel electrodes and 
is comparable to other EEG systems researchers use, and the consumer grade EPOC device, which uses 14 electrodes dampened with a salt water solution. There exists a large inter-subject variance in the ability to measure a clear SSVEP response. The ability to control the game depends more on the user, than the device that was used. During a public event where the EPOC device was used, $36 \%$ achieved good control, $52 \%$ found it challenging, $12 \%$ achieved no control.

4) The user can monitor the output of the classifier and modify its parameters in real time. The detection algorithm runs in real time in the background, which output was visible on the screen at all times. This allowed the users to judge the effect of their actions and compensate for the latency between looking at the stimulus and the formation of a detectable SSVEP signal. When too many false positives were produced, the assistant tuned the threshold values in real time, using the buttons at the bottom of the screen (see figure 1). The effect could immediately be seen on the classifier output.

In summary, we have developed a game that runs successfully on consumer grade hardware, allowing us to take it out of the lab more easily and present it during a public event. The feedback one receives during these events is different from the feedback during lab sessions, because the users only have a few minutes with the system and are easily bored or distracted. One of the major things we learned is that currently, the rules of the challenging, tactical game play take too long to explain for quick demonstrations. Even when the system could robustly classify the SSVEP response of the user, some users did not feel in control, because they did not fully understand the game rules. This could be overcome by gradually introducing new rules over time, instead of presenting the complete array of options all at once.

We have shown that SSVEPs can be detected robustly on the Emotiv EPOC, opening the way to commercial BCI games controlled by brain activity only. In the future, the other paradigms mentioned in the introduction, namely detection and classification of the the P300 potential and ERD/ERS, might be feasible with the EPOC as well, allowing for even more possibilities for new types of games.

\section{ACKNOWLEDGEMENTS}

The authors are grateful to Refet Firat Yazicioglu, Tom Torfs and Cris Van Hoof from imec Leuven, Belgium, for providing us with the wireless EEG system. MvV is sup- ported by the Belgian Fund for Scientific Research - Flanders (G.05809), AC and AR are supported by the Flemish Agency for Innovation through Science and Technology (IWT), NC by the Interuniversity Attraction Poles Programme - Belgian Science Policy (IUAP P6/29), and NVM by the Flemish Regional Ministry of Education (Belgium) (GOA 10/019). MMVH is supported by research grants received from the program Financing program (PFV/10/008) and the CREA Financing program (CREA/07/027) of the K.U.Leuven, the Belgian Fund for Scientific Research Flanders (G.0588.09), the Interuniversity Attraction Poles Programme - Belgian Science Policy (IUAP P6/29), the Flemish Regional Ministry of Education (Belgium) (GOA 10/019), and the European Commission (IST-2007-217077), and by the SWIFT prize of the King Baudouin Foundation of Belgium.

\section{REFERENCES}

[1] N. Birbaumer, "Breaking the silence: brain-computer interfaces (BCI) for communication and motor control." Psychophysiology, vol. 43, no. 6, pp. 517-532, 2006.

[2] A. Nijholt, B. Reuderink, and D. Oude Bos, "Turning shortcomings into challenges: Braincomputer interfaces for games," in Intelligent Technologies for Interactive Entertainment (INTETAIN), ser. Lecture Notes of the Institute for Computer Sciences, Social Informatics and Telecommunications Engineering, vol. 9, no. 2. Springer Berlin / Heidelberg, 2009, pp. 153-168.

[3] S. Hjelm and C. Browall, "Brainball - Using brain activity for cool competition," in Proceedings of NordiCHI, 2000, pp. 177-188.

[4] A. Nijholt, "BCI for Games: A 'State of the Art' Survey," in Entertainment Computing (ICEC 2008), ser. Lecture Notes in Computer Science, vol. 5309, no. 1. Springer Berlin / Heidelberg, 2009, pp. 225-228.

[5] A. Finke, A. Lenhardt, and H. Ritter, "The MindGame: a P300-based brain-computer interface game." Neural networks: the official journal of the International Neural Network Society, vol. 22, no. 9, pp. 1329-1333, 2009.

[6] M. W. Tangermann, M. Krauledat, K. Grzeska, M. Sagebaum, C. Vidaurre, and B. Blankertz, "Playing Pinball with non-invasive BCI," Advances in Neural Information Processing Systems, vol. 21, pp. 16411648, 2009.

[7] N. Chumerin, N. V. Manyakov, A. Combaz, A. Robben, M. V. Vliet, and M. M. V. H. Hulle, "Steady State Visual Evoked Potential based Computer Gaming - The Maze," in Intelligent Technologies for Interactive Entertainment (INTETAIN), 2011.

[8] M. a. Pastor, J. Artieda, J. Arbizu, M. Valencia, and J. C. Masdeu, "Human cerebral activation during steady-state visual-evoked responses." The Journal of neuroscience: the official journal of the Society for Neuroscience, vol. 23, no. 37, pp. 11621-11727, 2003.

[9] O. Friman, I. Volosyak, and A. Gräser, "Multiple channel detection of steady-state visual evoked potentials for brain-computer interfaces." IEEE transactions on bio-medical engineering, vol. 54, no. 4, pp. 742 $750,2007$.

[10] A. Luo and T. J. Sullivan, "A user-friendly SSVEP-based brain-computer interface using a time-domain classifier." Journal of neural engineering, vol. 7, no. 2, p. 26010, 2010.

[11] J. Cardoso and A. Souloumiac, "Blind beamforming for non-gaussian signals," IEEE Proceedings for Radar and Signal Processing, vol. 140, no. 6, pp. 362-370, 1993. 